\title{
IDENTIFYING OPINION LEADERS USING SOCIAL NETWORK ANALYSIS, A STUDY IN AN EGYPTIAN VILLAGE
}

\author{
Mohamed Mohamed Mohamed Abdel-Ghany, Assistant Professor
}

Assiut University, Assiut, Egypt

Phone: +201003718860, E-mail: abdelghany18@yahoo.com

Received April 3, 2012

\begin{abstract}
This research used four approaches to identify opinion leaders in two networks in an Egyptian Village. One approach was used for identifying single opinion leaders (network centrality measures), and three approaches were used to identify sets of key players (group centrality, network efficiency, and KPP-Pos \& KPP-Neg). Social network analysis software (UCINET 6) was used for individual \& group centrality measures. The study used the key player software to identify sets of key players under KPP-Pos \& KPPNeg. The study identified different opinion leaders using the four different techniques. This research gives an opportunity for further work on a comprehensive approach to identify key players in social networks to overcome the problem of identifying different leaders by different techniques without an absolute answer for the question concerning which technique will capture the best leaders.
\end{abstract}

\section{KEY WORDS}

Opinion Leaders; Key Players; Network Analysis; Centrality; Network Efficiency; Egypt.

Studying influence in social networks is an important topic that has attracted the attention of a variety of researchers in different domains. People often seek the opinion and advice of their peers regarding various decisions. This behavior gives rise to a certain set of individuals in the social network, referred to as influentials or opinion leaders, who have a huge impact on other people's opinions, actions and behavior (Sharara et al., 2011). Considering the importance of opinion leadership in social networks, studies show that different titles (such as opinion leaders, influential people, and key players) are used to refer to the influential group in social networks (Momtaz et al., 2011).

Social network analysis is the process of applying analysis techniques to a social network to answer specific questions about that network (McGuire, 2011). A natural question to ask is thus: can network analysis be used as an identifier of opinion leaders? In terms of identifying opinion leaders, Kim (2007) stated that social network analysis has its unique advantages, distinguished from other methods. Social network analysis scrutinizes relationships with the directions of the relationships. SNA also generates various social network maps showing communicative relationships among members of a social system. This visualization enables researchers to identify opinion leaders in a social system. Third, social network analysis provides more accurate and realistic analyses, because the data are constructed by contacting most of, or even all of, a population in a social group.

The discovery of key players in social networks can be done using different methodologies for both single key players and sets of key players. This research endeavors particularly to use different approaches that are applied to find single and sets of opinion leaders in a village in Assiut Governorate, Egypt. In the following, after an outline of opinion leaders, methods for discovering key players are demonstrated, later, after methodology and results, major conclusions are derived.

\section{LITERATURE REVIEW}

Opinion leaders: definition, characteristics, and influence. In 1944, professor Lazarsfeld, in his researches, has found that the public communication does not directly flow to the mass but be interpreted firstly by opinion leaders and then reach the common people. The process is: the mass medium $\rightarrow$ the opinion leaders $\rightarrow$ the common people. That is the so-called "two step flow communication". The main contributions of this theory are: (1) Information can be transferred not only by medium but also by interpersonal com- 
munication network. In other words, people can obtain information by two channels or any of the two; (2) There is an interface between the medium and the interpersonal communication network, And the interface is the opinion leader; (3) The influences of opinion leaders and interpersonal communication network on information communication and individuals' decision are far larger than that of the mass medium (Zhang\& Dong, 2008)

Before making decisions individuals often seek to reinforce their opinions through "consensual validation" with certain others. Among these "certain others" are individuals who exert an unequal amount of influence on the decisions of others: they are called "opinion leaders" (Rogers \& Cartano, 1962). Opinion leadership refers to "the degree to which an individual is able to influence other individuals' attitudes or behavior informally in a desired way with relative frequency" (Rogers, 1983: 271), and opinion leaders are people who influence the opinions, attitudes, beliefs, motivations, and behaviors of others (Valente \& Pumpuang, 2007).

In contrast with their followers, opinion leaders have these characteristics in general: (1) They can obtain information by more channels and have rich life experiences. They are knowledgeable and professional in one specialty; They can contact with innovation agencies frequently; (2) They prefer to take part in formal or informal social activities. They have a wide social relationship and connect with the public closely; (3) They have farreaching insight, innovative spirit; energetic thoughts and they would like to accept new things (Zhang \& Dong, 2008). More specifically, through decades of studies, Rogers (2003: 316-318) identified seven generalizable characteristics of opinion leaders: (1) greater exposure to mass media; (2) more cosmopolite; (3) greater contact with change agents; (4) greater social participation; (5) higher socioeconomic status; (6) more innovative; (7) more innovative.

Opinion leaders can influence their peers in several ways. Chau and Hui (1998) identify three main ways in which opinion leaders 'exert an unequal amount of influence on the decisions of others'. They are '(1) acting as role models who inspire imitation; (2) spreading information via word of mouth; and (3) giving advice and verbal direction for search, purchase, and use'. The measurement of opinion leadership is usually a very tedious process.

Methods for Discovering Key Players in social networks. The discovery of single key players in social networks is commonly done using some of the centrality measures employed in social network analysis. However, few methods, aimed at discovering sets of key players, have been proposed in the literature (Arroyo,2010).

In order to identify single opinion leaders by using social network analysis, the most important and frequently used concept is network centrality. Network centrality measures structural importance of actors and indicates which actors can be regarded as those who are in the center of networks(Borgatti, 2006). Network centrality has been mainly calculated by using three measures which are degree, betweenness, and closeness: (1) degree - measures how many direct connections an individual (node) has; (2) betweeness - measures how much an individual controls communication flows between/among other individuals or different communication networks; and (3) closeness - measures how quickly an individual can access all other individuals via a minimum of steps. In opinion leadership studies, individuals who have higher degrees in one of these measures are conventionally regarded as those who would be opinion leaders (Kim et al., 2007).

In terms of the methods used to discover sets of key players, The first method is concerned about group centrality. The concept of centrality has been applied not only to single individuals within a network but also to groups of individuals. In (Everett\& Borgatti,2005), measures for degree centrality, closeness, and betweenness are defined for a group. Using these measures, groups having high centrality will be the key players.

Another approach to find key players is based on measuring the communication efficiency of a network. The efficiency $\mathrm{E}$ of a network $\mathrm{G}$ was defined in Eq.1:

$$
E(G)=\frac{\sum_{i \neq j \in G} \varepsilon_{i j}}{N(N-1)}=\frac{1}{N(N-1)} \sum_{i \neq j \in G} \frac{1}{d_{i j}}
$$

where $\mathrm{N}$ is the number of nodes in graph $\mathrm{G}$ and "ij is the communication efficiency, which is proportional to the inverse of dij (the shortest path length between two nodes $i ; j$ ). The equation calculates all shortest paths between all pairs of nodes normalized by the number of all possible paths that will be contained in a fully connected graph consisting of $\mathrm{N}$ nodes. The method essentially consists in removing nodes one by one, recalculating the drop in network efficiency every time. These nodes that produce the largest impact in reducing the overall efficiency of a network are selected as the key players (Latora \& Marchiori, 2004). 
Third approach to discover sets of key players, proposed by Borgatti (2006), consists in selecting simultaneously $\mathrm{k}$ players via combinatorial optimization. In that work, Borgatti defines two problems related to discovering sets of key players as follow. The Key Player Problem Positive (KPPPos) consists of identifying these k-players that could be used as seeds in diffusing optimally some information on the network. The Key Player Problem Negative (KPP-Neg) goal consists of identifying those k-players that, if removed, will disrupt or fragment the network. A more formal definition of the two problems is "Given a social network, find a set of k nodes (called a kp-set of order k) such that, 1. (KPP-Neg) Removing the kp-set would result in a residual network with the least possible cohesion. 2. (KPP-Pos) The kp-set is maximally connected to all other nodes." Borgatti found that off-the-shelf centrality measures are not appropriate for the task of discovering sets of key players as defined by KPP-Pos and KPP-Neg problems.

\section{METHODOLOGY}

ELKORDY village - Assiut Governorate was chosen to be a place of this study. Data were collected from all farmers of the village (72 farmers) by questionnaire. As examples of how the above mentioned methods of identifying opinion leaders work in practice, the study presents an application to two networks, the first is about the opinion leadership concerning the agricultural is- sues and the other one is about the opinion leadership concerning the cattle issues. The survey asked farmers to name one farmer whom they talk with or whom they get advice from about the agricultural issues \& the cattle issues. Social network analysis software (UCINET 6) was used for individual \& group centrality measures, and NetDraw for drawing the studied networks (Borgatti et al., 2002). The study used the key player software to identify sets of key players under KPP-Pos \& KPP-Neg (Borgatti, 2008).

\section{RESULTS}

Network 1: opinion leadership concerning the agricultural issues. The first application of the methods of identifying opinion leaders is the network of opinion leadership concerning the agricultural issues (Figure1). The survey asked farmers to name one farmer whom they talk with or whom they get advice from about agricultural issues. In order to identify single opinion leaders by using social network analysis, the study used network centrality measures for degree centrality, closeness, and betweenness. Table 1 shows the single opinion leaders inside network 1 , they are: $45,30,19,21,22$ and 68 with respect to degree centrality, they are: $24,21,42,45,68$ and 19 with respect to Closeness centrality, they are: $21,24,68,50,55$ and 45 with respect to Betweenness centrality.

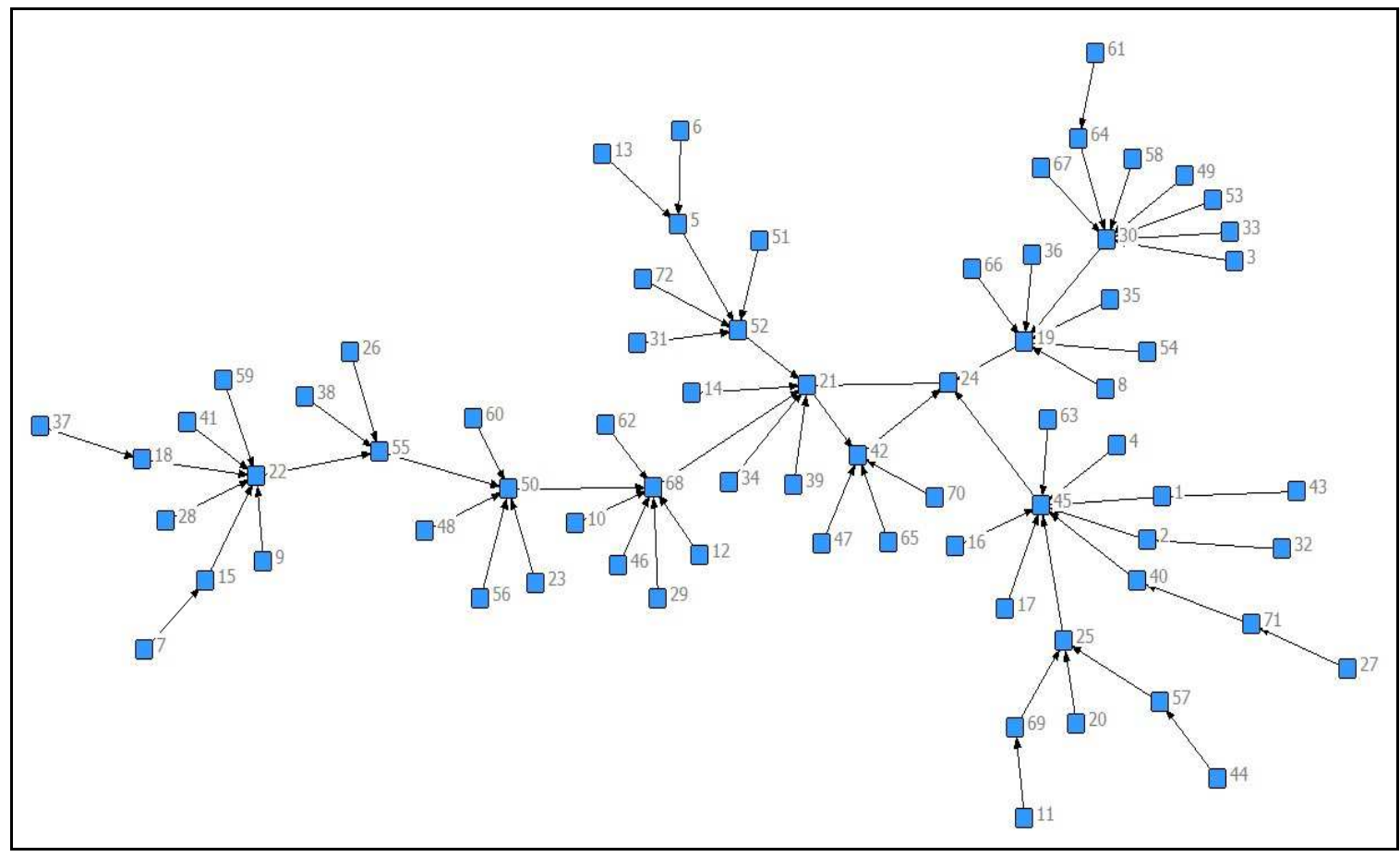

Figure 1-Network 1: opinion leadership concerning the agricultural issues 
Table 1 - Network centrality measures for single opinion leaders inside network 1

\begin{tabular}{|c|c|c|c|c|c|}
\hline No. & Degree & No. & Closeness & No. & Betweenness \\
\hline 45 & 9 & 24 & 34.63 & 21 & 100 \\
\hline 30 & 8 & 21 & 33.81 & 24 & 70 \\
\hline 19 & 7 & 42 & 30.47 & 68 & 66 \\
\hline 21 & 7 & 45 & 29.46 & 50 & 64 \\
\hline 22 & 7 & 68 & 29.10 & 55 & 55 \\
\hline 68 & 7 & 19 & 28.74 & 45 & 51 \\
\hline
\end{tabular}

It's time to turn attention to identify the sets of key players inside network 1 . The first approach depends on group centrality, using UCINET to calculate group centrality measures, the key players with respect to group centrality are: $30,42,45,50$ and 68 . The second approach is based on measuring the communication efficiency of the network using the above mentioned Eq.1. The study deactivated one by one of the 72 nodes and calculated the efficiency of the new network (E) and the drop of the efficiency caused (DE /E). In Table 2, the study reported the most important nodes, the numbers reported in table 2 indicate that farmers 21, 42 and 24 are three key nodes, in fact their damage would reduce more than $50 \%$ of the efficiency of the whole network.

The third approach is related to Key Player Problem Positive (KPP-Pos) \& Key Player Problem Negative (KPP-Neg). Fig.2. shows the key players with respect to KPP-Pos. The kp-set is maximally connected to all other nodes, they are: $21,22,25,30,40,45,50$ and 52. Fig. 3. represents the set of key players with respect to KPP-Neg. It is the identification of key players for fragmenting the network by removing the key nodes. The nodes identified are: 19, 21, 22, 45 and 50. If those k-players removed, this will disrupt 0.958 of the network.

Table 2 - Effect of the deactivation of a node inside network 1

\begin{tabular}{|c|c|c|}
\hline Removed node & E $(\mathrm{G}-$ node $)$ & $\mathrm{DE} / \mathrm{E}$ \\
\hline 21 & 0.0641 & 0.673 \\
\hline 42 & 0.0933 & 0.524 \\
\hline 24 & 0.0959 & 0.511 \\
\hline 68 & 0.1066 & 0.457 \\
\hline 50 & 0.1114 & 0.432 \\
\hline 55 & 0.1213 & 0.382 \\
\hline 22 & 0.1498 & 0.237 \\
\hline 30 & 0.1557 & 0.207 \\
\hline- & - & -
\end{tabular}

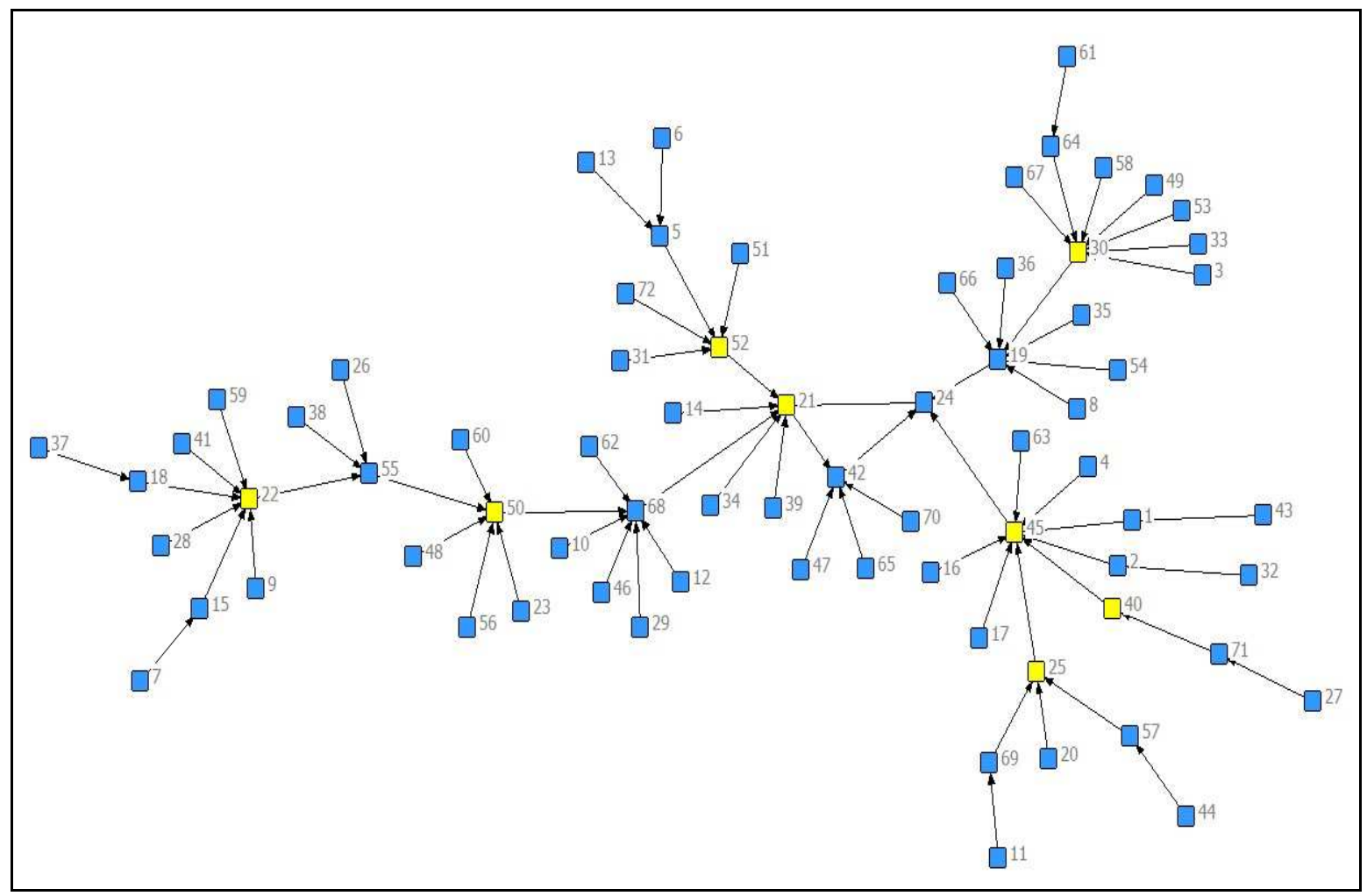

Figure 2 - A Set of Key Players (yellow in color) under KPP-Pos inside network 1 


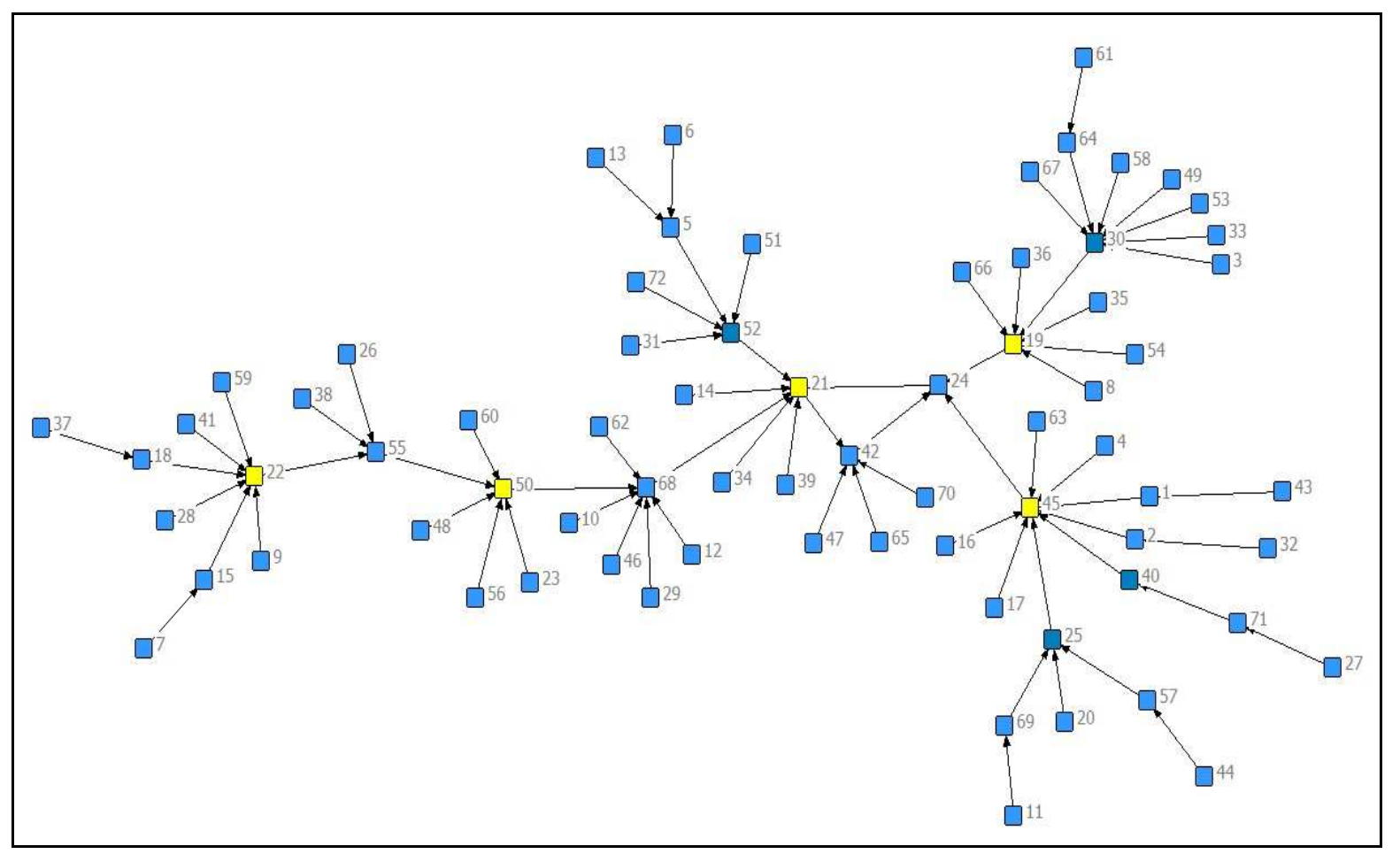

Figure 3 - A Set of Key Players (yellow in color) under KPP-Neg inside network 1

Table 3 presents a summary of opinion leaders identified by different methods inside network 1. It's obvious that there's no such an agreement among the mentioned techniques about who are the opinion leaders inside network 1 , although there are some repeated nodes within these techniques.

Table 3 - Opinion leaders identified by different methods inside network 1

\begin{tabular}{|c|c|}
\hline Methods & Key Players \\
\hline Degree Centrality & $45,30,19,21,22,68$ \\
\hline Closeness Centrality & $24,21,42,45,68,19$ \\
\hline Betweenness Centrality & $21,24,68,50,55,45$ \\
\hline Group Centrality & $30,42,45,50,68$ \\
\hline Network Efficiency & $21,42,24$ \\
\hline KPP-Pos & $21,22,25,30,40,45,50,52$ \\
\hline KPP-Neg & $19,21,22,45,50$ \\
\hline
\end{tabular}

Network 2: opinion leadership concerning the cattle issues. The second application of the methods of identifying opinion leaders is the network of opinion leadership concerning the cattle issues (Figure4). The survey asked farmers to name one farmer whom they talk with or whom they get advice from about cattle issues. In order to identify single opinion leaders by using social network analysis, the study used network centrality measures for degree centrality, closeness, and betweenness. Table 3 reveals the single opinion leaders inside network 2,they are:
$17,50,30,45,20$ and 52 with respect to degree centrality, they are: $30,45,52,50,42$ and 64 with respect to Closeness centrality, they are: $50,30,52,20,45$ and 39 with respect to Betweenness centrality.

The other side of network 2 is related to identifying the sets of key players. The first approach counts on group centrality, key players with respect to group centrality are: $17,45,50,55$ and 68. The second approach is based on measuring the communication efficiency of the network using the above mentioned Eq.1. The study deactivated one by one of the 72 nodes and calculated the efficiency of the new network (E) and the drop of the efficiency caused (DE /E). In Table 4, the study represented the most important nodes, the numbers reported in table indicate that farmers 52 and 50 are two key nodes, in fact their damage would reduce more than $50 \%$ of the efficiency of the whole network.

Table 4 - Network centrality measures for single opinion leaders inside network 2

\begin{tabular}{|c|c|c|c|c|c|}
\hline No. & Degree & No. & Closeness & No. & Betweenness \\
\hline 17 & 9 & 30 & 32.27 & 50 & 88 \\
\hline 50 & 8 & 45 & 30.34 & 30 & 84 \\
\hline 30 & 7 & 52 & 30.08 & 52 & 81 \\
\hline 45 & 7 & 50 & 27.10 & 20 & 65 \\
\hline 20 & 6 & 42 & 25.72 & 45 & 61 \\
\hline 52 & 6 & 64 & 24.83 & 39 & 30 \\
\hline
\end{tabular}




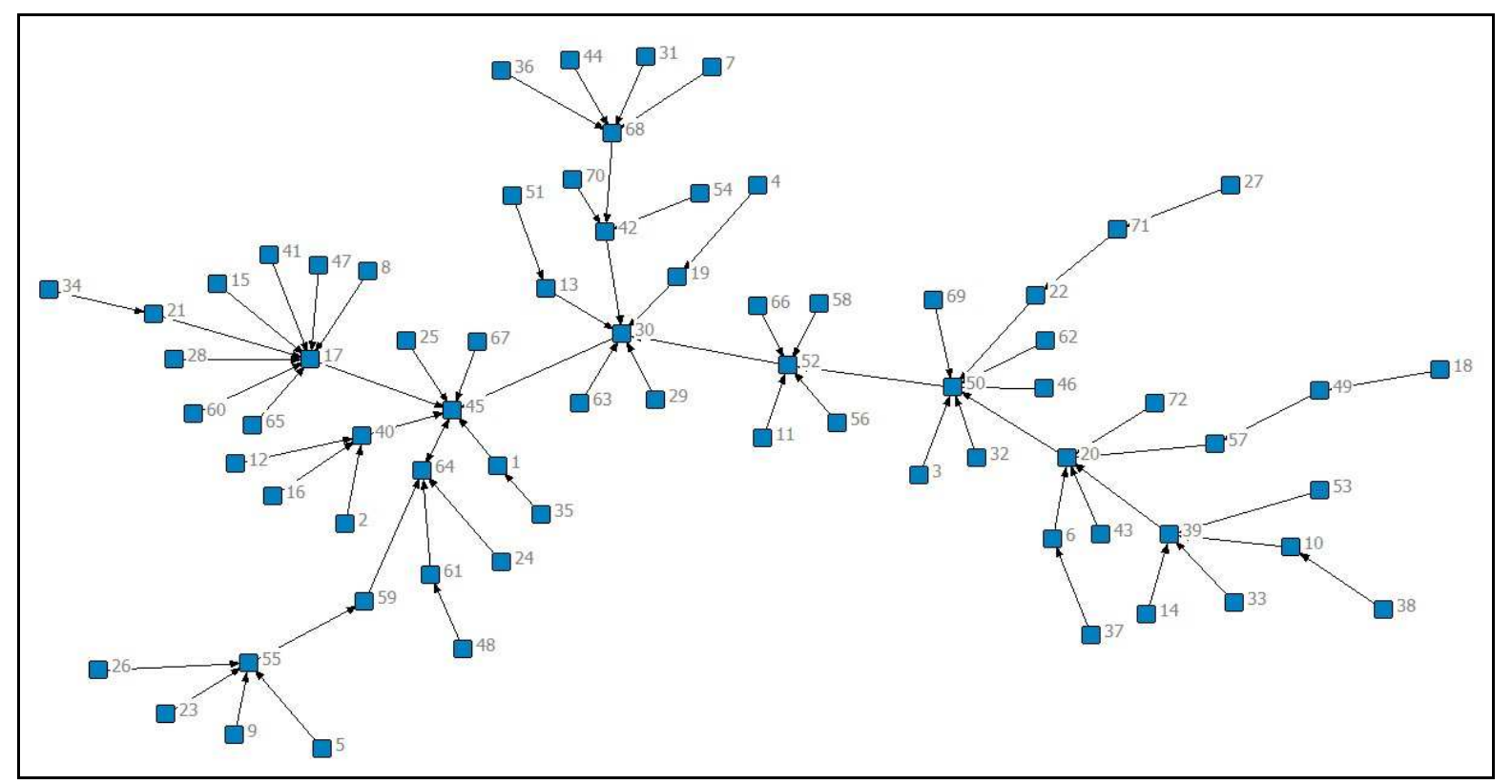

Figure 4 - Network2: opinion leadership concerning the cattle issues

The third approach is connected with Key Player Problem Positive (KPP-Pos) \& Key Player Problem Negative (KPP-Neg). Fig.5. shows the key players with respect to KPP-Pos. The kp-set is maximally connected to all other nodes, they are: $17,20,22,30,39,42,45,57,59$ and 64 .

Fig. 6 represents the set of key players with respect to KPP-Neg. It is the identification of key players for disrupting or fragmenting the network by removing the key nodes. The nodes identified are: 17, 20, 30, 45 and 50. If those k-players removed, this will fragment 0.955 of the network.
Table 5 - Effect of the deactivation of a node inside network 2

\begin{tabular}{|c|c|c|}
\hline Removed node & E $(\mathrm{G}$ - node & $\mathrm{DE} / \mathrm{E}$ \\
\hline 52 & 0.1120 & 0.630 \\
\hline 50 & 0.1239 & 0.591 \\
\hline 20 & 0.1788 & 0.410 \\
\hline 45 & 0.1895 & 0.375 \\
\hline 55 & 0.2398 & 0.209 \\
\hline 64 & 0.2426 & 0.200 \\
\hline$----o 5$ &
\end{tabular}

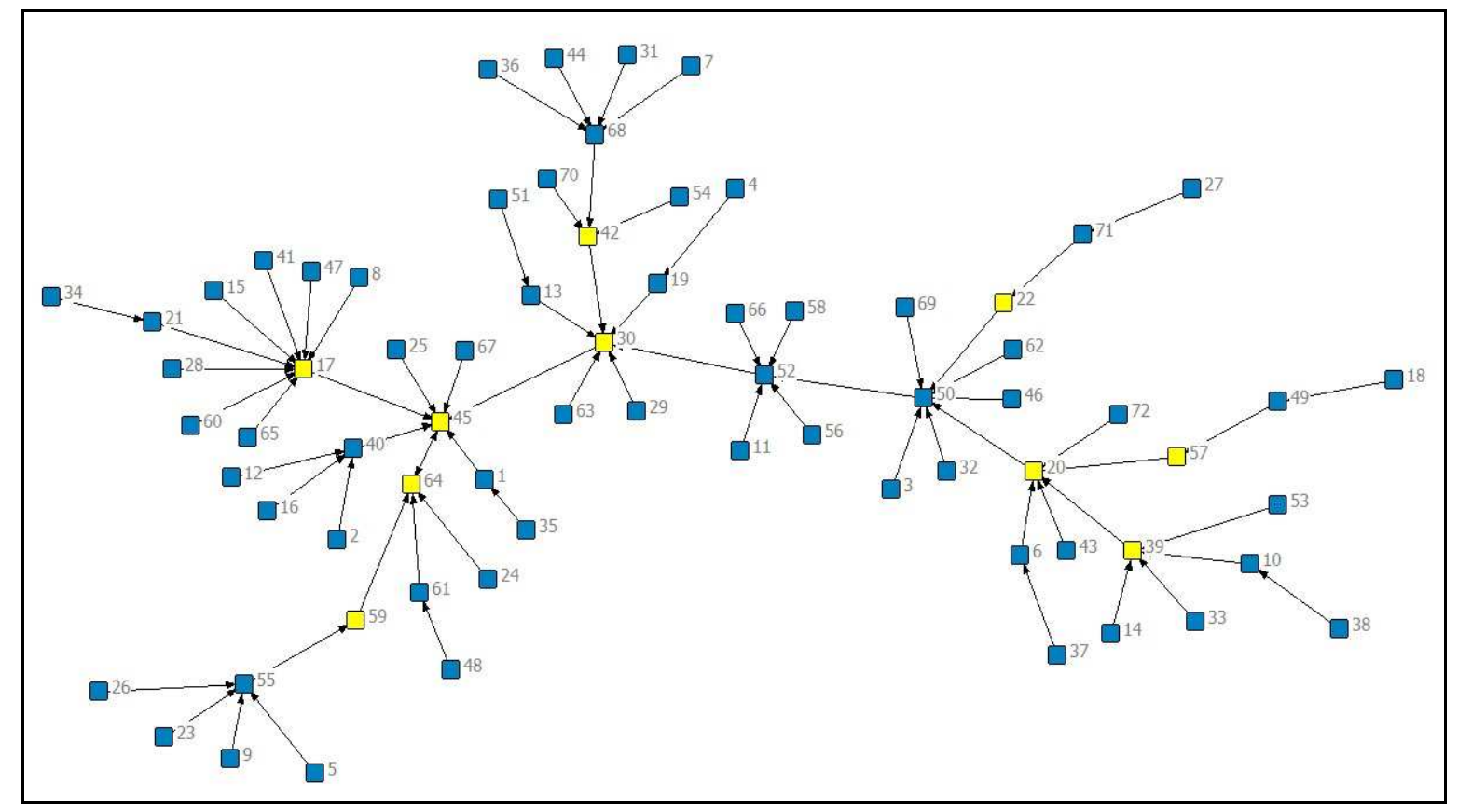

Figure 5 - A Set of Key Players (yellow in color) under KPP-Pos inside network 2 


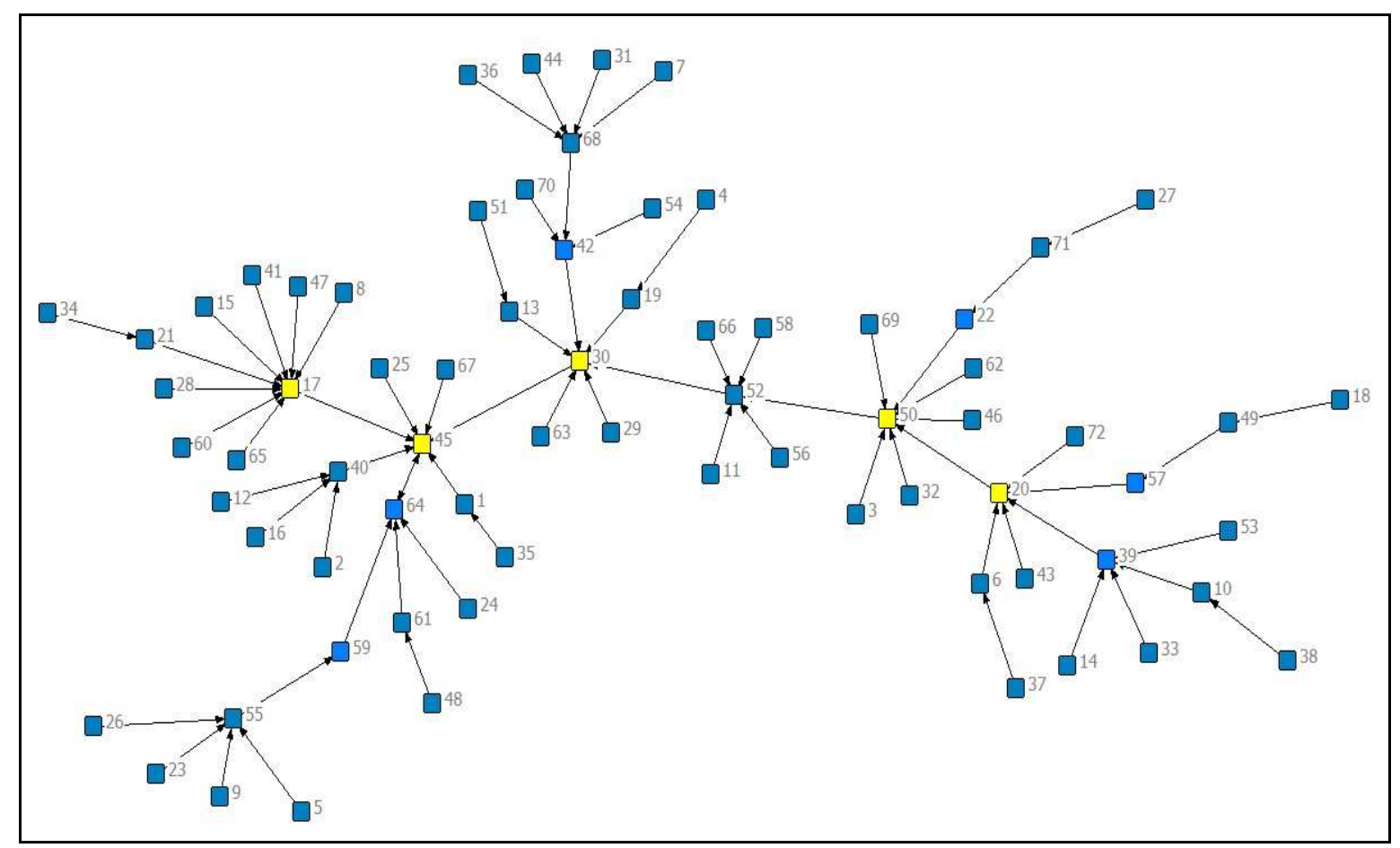

Figure 6 - A Set of Key Players (yellow in color) under KPP-Neg inside network 2

Table 6 presents a summary of opinion leaders identified by different methods inside network 2 .

Table 6 - Opinion leaders identified by different methods inside network 2

\begin{tabular}{|l|l|}
\hline Method & Key Players \\
\hline Degree Centrality & $17,50,30,45,20,52$ \\
\hline Closeness Centrality & $30,45,52,50,42,64$ \\
\hline Betweenness Centrality & $50,30,52,20,45,39$ \\
\hline Groups Centrality & $17,45,50,55,68$ \\
\hline Network Efficiency & 52,50 \\
\hline KPP-Pos & $17,20,22,30,39,42,45,57,59,64$ \\
\hline KPP-Neg & $17,20,30,45,50$ \\
\hline
\end{tabular}

As in network 1, It's manifest that there's no such an agreement between the stated techniques about who are the opinion leaders inside network 2 , despite the fact that there are some repeated nodes within these techniques.

\section{CONCLUSION}

Considering the importance of opinion leadership in social networks, Identifying importance of nodes in a network has been an active problem in many research areas. Key players can be identified using different methods of social network analysis. This research used four techniques to identify opinion leaders in two networks.

It is obvious that different measures for identifying opinion leaders result in different nodes. That's because these different measures capture different aspects of what it means for a node to be "importance" to the network. The two networks contain nodes that in spite of not having a high degree, have in fact a greater impact in disrupting the network structure when removed. Based on the general findings of this research, it was revealed that different leaders were identified for different situations (agricultural issues network, cattle issues network). Thus, the idea of situation based leadership can be accepted at the level of the studied networks.

A possible extension to this study is to provide a comprehensive approach to identify key players in a social network that are located to influence, intercept, strengthen or disrupt flow among network members. This approach includes somehow the above mentioned techniques and takes into account both the direct and indirect links in the network. At that time, this approach will overcome the problem of identifying different leaders without an absolute answer for the question concerning which method will capture the best leaders. 


\section{REFERENCES}

Arroyo, D. (2010). "Discovering Sets of Key Players in Social Networks". Book Chapter in Computational Social Networks Analysis, Trends, Tools and Research Advances published by Springer in the Computer and Communication Networks Series.

Borgatti, S. (2006). Identifying sets of key players in a network. Comput Math Organ Theory, 12(1):21-34.

Borgatti, S. (2008). Key Player Program, Harvard, MA: Analytic Technologies.

Borgatti, S. Everett, M. \& Freeman, L. (2002). Ucinet for Windows: Software for Social Network Analysis. Harvard, MA: Analytic Technologies.

Chau, P. \& Hui, K.( 1998). 'Identifying early adopters of new IT products: A case for Windows 95', Information \& Management, 33:225-230.

Everett, M. \& Borgatti, S. (2005). "Extending centrality". Book Chapter in Models and methods in social network analysis published by Cambridge University Press.

Kim, D; Chitnis, K; Vasanti, P. \& Singhal, A. (2007). Opinion Leadership Networks and Diffusion of e-Choupal in Indian Villages, Journal of Creative Communications, 2 (3): 345-360.

Kim, D. (2007). Identifying Opinion Leaders by Using Social Network Analysis: A Synthesis of Opinion Leadership Data Collection Methods and Instruments, $\mathrm{PhD}$ thesis, the faculty of the Scripps College of Communication of Ohio University.

Latora ,V \& Marchiori, M. (2004). How the science of complex networks can help developing strategies against terrorism. Chaos, Solitons and Fractals 20(1): 69-75.

McGuire, R. (2011). Weighted Key Player Problem for Social Network Analysis, Master Thesis, Department of Operational Sciences, Graduate School of Engineering and Management, Air Force Institute of Technology, Air University, Ohio, USA.

Momtaz, M., Aghaie, A. \& Alizadeh, S. (2011) Identifying Opinion Leaders for Marketing by Analyzing Online Social Networks, International Journal of Virtual Communities and Social Networking, 3(1), 43-59.
Rogers, E. \& Cartano, D. (1962). Methods of Measuring Opinion Leadership, The Public Opinion Quarterly, 26(3): 435-441.

Rogers, E. (1983). Diffusion of Innovations, 3rd Edition, Free Press, New York.

Rogers, E. (2003). Diffusion of innovations, 5th Edition, Free Press, New York.

Sharara, H; Getoor, L \& Norton, M.(2011) Active Surveying: A Probabilistic Approach for Identifying Key Opinion Leaders, Proceedings of the Twenty-Second International Joint Conference on Artificial Intelligence, Barcelona, Catalonia, Spain, 16-22 July.

Valente, T. \& Pumpuang, P.(2007). Identifying opinion leaders to promote behavior change, Health Educ. Behav. 34 (6): 881896.

Zhang, X. \& Dong, D.(2008). Ways of Identifying the Opinion Leaders in Virtual Communities, International Journal of Business and Management, 3(7): 21-27. 